\title{
Diabetes mellitus: an important risk factor for reactivation of tuberculosis
}

\author{
Ernesto Solá', Carmen Rivera', Michelle Mangual', José Martinez', Kelvin Rivera² \\ and Ricardo Fernandez ${ }^{2}$
}

1Endocrinology Department, San Juan City Hospital, San Juan, Puerto Rico, USA and

2Pulmonary Medicine Department, San Juan City Hospital, San Juan, Puerto Rico, USA
Correspondence

should be addressed

to E Solá

Email

ernestojsola@gmail.com

\section{Summary}

Diabetes mellitus was identified as a risk factor for developing tuberculosis (TB) infection, and relapse after therapy. The risk of acquiring TB is described as comparable to that of HIV population. The fact that diabetics are $3 \times$ times more prone to develop pulmonary TB than nondiabetics cannot be overlooked. With DM recognized as global epidemic, and TB affecting one-third of the world population, physicians must remain vigilant. We present a 45 -year-old woman born in Dominican Republic (DR), with 10-year history of T2DM treated with metformin, arrived to our Urgency Room complaining of dry cough for the past 3 months. Interview unveiled unintentional $15 \mathrm{lbs}$ weight loss, night sweats, occasional unquantified fever, and general malaise but denied bloody sputum. She traveled to DR 2 years before, with no known ill exposure. Physical examination showed a thin body habitus, otherwise well appearing woman with stable vital signs, presenting solely right middle lung field ronchi. LDH, ESR, hsCRP and Hg A1C were elevated. Imaging revealed a right middle lobe cavitation. Sputum for AFB disclosed active pulmonary TB. Our case portrays that the consideration of TB as differential diagnosis in diabetics should be exercised with the same strength, as it is undertaken during the evaluation of HIV patients with lung cavitation. Inability to recognize TB will endanger the patient, hospital dwellers and staff, and perpetuate this global public health menace.

\section{Learning points:}

- Diabetes mellitus should be considered an important risk factor for the reactivation of pulmonary tuberculosis.

- High clinical suspicious should be taken into consideration as radiological findings for pulmonary tuberculosis in patients with diabetes mellitus may be atypical, involving middle and lower lobes.

- Inability to recognize pulmonary tuberculosis will endanger the patient, hospital dwellers and staff, and perpetuate this global public health menace.

\section{Background}

Diabetes mellitus (DM) is recognized as a risk factor for the reactivation of tuberculosis (TB) infection, and relapse after therapy. Strikingly in Hispanics, the risk of DM patients of acquiring TB (25.2\%) is described as comparable to that of HIV population (25.5\%), taking into consideration that this population is known to have a higher diabetes prevalence when compared with other demographics (1). Abnormal cytokine expression in diabetic patients is proposed as underlying mechanism, yet to be fully understood (2). Still, the fact that diabetics are three times more prone to develop pulmonary TB than nondiabetics and may present with atypical imaging findings, irrespective of background incidence or geographic region, cannot be overlooked $(2,3)$. With DM recognized as global epidemic, and TB affecting one-third of the world population, physicians must remain vigilant (3). 


\section{Clinical case}

A 45-year-old woman born in Dominican Republic (DR), with 10-year history of T2DM treated with metformin, arrived to our Urgency Room complaining of dry cough for the past 3 months. Interview also revealed unintentional $15 \mathrm{lbs}$. weight loss, night sweats, occasional unquantified fever and general malaise but denied bloody sputum. She traveled to DR 2 years before without any ill exposure. Surgical history was remarkable for TAH due to pelvic cancer. Physical examination showed a thin body habitus, otherwise well appearing woman with stable vital signs, presenting solely right middle lung field ronchi up on auscultation. Laboratories yielded no leukocytosis or monocytosis. Blood cultures, HIV and PPD tests were all negative. However, LDH (997 units/L), ESR $(80 \mathrm{~mm} / \mathrm{h})$, hsCRP $(71.2 \mathrm{mg} / \mathrm{L})$ and HbA1C $(12.4$ - avg blood glucose $309 \mathrm{mg} / \mathrm{dL}$ ) were elevated. Imaging showed a right upper lobe cavitation suspicious for malignancy vs infection (Figs 1 and 2). Positive sputum for AFB disclosed active pulmonary TB in our patient, prompting therapy. Hospital course complicated with hyperglycemia despite frequent insulin dose escalation even after clearance of AFB sputum.

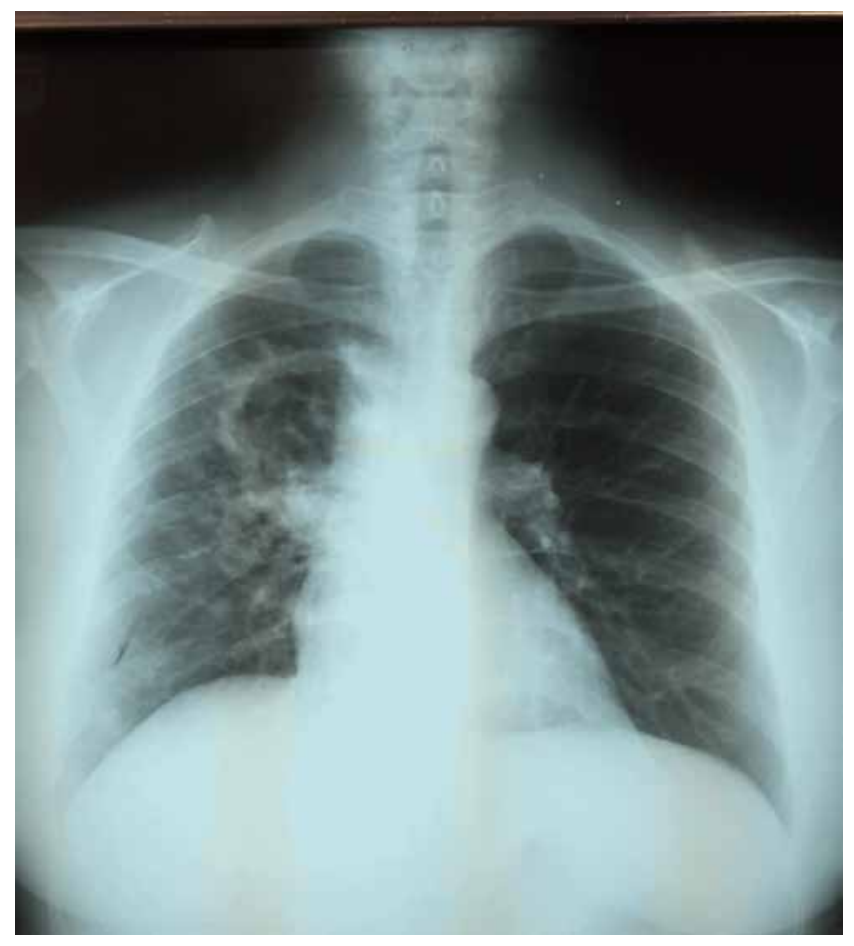

Figure 1

PA chest $x$-ray; right lung cavitary lesion.

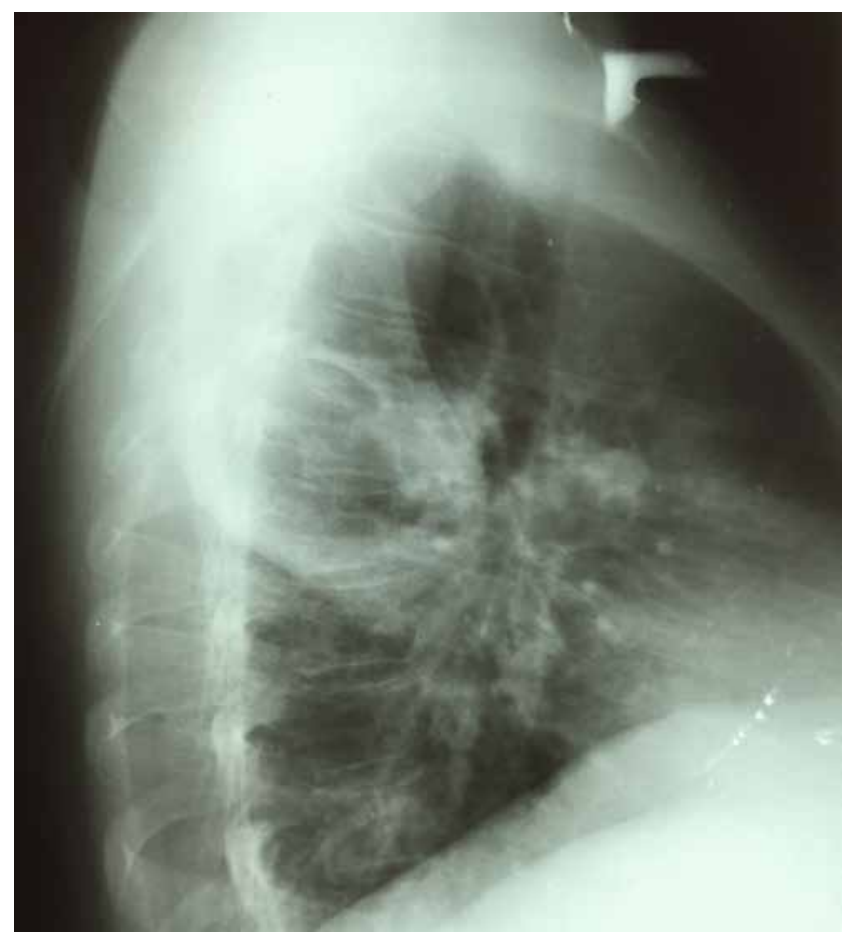

Figure 2

Lateral chest x-ray; right upper lung cavitary lesion.

\section{Investigation}

- CXR right lung cavitary lesion with fluid levels suspicions for lung abscess.

- LDH (997 units/L), ESR ( $80 \mathrm{~mm} / \mathrm{h})$, hsCRP $(71.2 \mathrm{mg} / \mathrm{L})$ and HbA1C (12.4 - avg blood glucose $309 \mathrm{mg} / \mathrm{dL}$ ) were elevated.

- Gram stain positive for AFB.

- Sputum culture positive for Mycobacterium tuberculosis.

\section{Treatment}

The patient was started on four-drug regimen: ethambutol, rifampin, isoniazid and pyrazinamide, and deescalated to rifampin and isoniazid once susceptibility was available for 6 months total. In addition to TB therapy, patient was treated with regular and basal Insulin requiring considerable doses to achieve DM control.

\section{Outcome and follow-up}

Patient was treated successfully and evidenced by culturenegative samples for Mycobacterium tuberculosis and interval resolution of cavitation on CXR. Additionally, insulin doses were reduced episodically during hospitalization and switched to oral therapy once discharged. 


\section{Discussion}

Diabetes is an important risk factor to consider in the evaluation of a patient with suspected pulmonary tuberculosis and even more because most patients can be overlooked and the diagnosis of TB could be missed (1). Several atypical clinical presentations as well as radiographic imaging can differ among nondiabetic and diabetic patients with pulmonary tuberculosis that need to be understood in order to consider this serious infectious process in an otherwise 'low-risk' diabetic patient $(3,4)$.

DM may increase susceptibility for pulmonary TB via multiple mechanisms. Those directly related to hyperglycemia and cellular insulinopenia and the indirect effect on macrophages and lymphocytes function, leading to diminished ability to contain the TB. DM is known to affect chemotaxis, phagocytosis, activation and antigen presentation by phagocytes in response to TB. Chemotaxis of monocytes is impaired, and this defect does not improve with insulin. Studies demonstrated that DM predisposes a patient to infections in which cell-mediated immunity has a pivotal role, such as TB $(4,5)$.

In terms of the incidence, it is known that DM is a moderate to strong risk factor for the development of active TB. There is a 3.1 chance to have TB in DM patients compared with non-DM populations $(3,6)$. In the Hispanic population among 25-54 years old, the risk for TB attributable to DM was $25 \%$, equivalent to that of HIV population. However, statistical studies have shown that the prevalence of DM is greater among Hispanics (up to $18.3 \%)$ compared with Hispanics with HIV (17\%) (7). Among insulin-dependent DM (IDDM) patients, there is an increased risk for developing acute TB compared with noninsulin-dependent DM population and that $\mathrm{HbA1C}$ greater than $7 \%$ has a three times increased hazard of active $\mathrm{TB}$ compared with those with HbA1C of less than $7 \%(4)$.

Radiological findings of TB in patients with DM not always present with the typical apical lesions seen in general population. Pulmonary TB in DM patients may present with an atypical radiological pattern and distribution particularly lower-lung involvement $(4,6)$. Clinically, it is important because lower lobe TB may be misdiagnosed as community-acquired pneumonia or even lung cancer. Also, patients with pulmonary TB that do not have upper lobe involvement are less likely to have positive sputum smears; therefore, other diagnostic methods for pulmonary TB should be considered such as bronchoalveolar lavage and transbroncheal biopsy, among others $(4,8)$. Clinicians should be aware of this atypical presentation when dealing with suspected $\mathrm{TB}$ on diabetic patients in order to make a precise diagnosis.

As per $\mathrm{TB}$ treatment, time to negative sputum cultures is longer among DM patients compared with control. In terms of the drugs used to treat TB, they can worsen glycemic control in patients with DM. Rifampin is a powerful inducer of host cytochrome P450 and, therefore, on IDDM patients; insulin requirements may increase during Rifampin therapy. Oral hypoglycemic drugs such as sulfonylureas are also affected by rifampin metabolism and could cause hyperglycemia making the whole process a vicious cycle between illness, body metabolism, diabetes mellitus and its respective treatments one affecting another (4).

Our case portrays that the consideration of TB as differential diagnosis in diabetics should be exercised with the same strength, as it is undertaken during the evaluation of HIV patients. Inability to recognize TB will endanger the patient, hospital dwellers and staff, and perpetuate this global public health menace.

\section{Declaration of interes}

The authors declare that there is no conflict of interest that could be perceived as prejudicing the impartiality of this case report.

\section{Funding}

This case report did not receive any specific grant from any funding agency in the public, commercial or not-for-profit sector.

\section{Patient consent}

A written informed consent was obtained from the patient for publication of the case.

\section{Author contribution statement}

Dr Kelvin Rivera was involved in articles review regarding diabetes and tuberculosis. Dr Carmen Rivera was involved in the out-patient care as endocrinologist after discharge. Dr Michelle Mangual is an endocrinologist who was involved in the patient care as consultant. Dr José Martinez is an endocrinologist who was involved in the patient care as a consultant. Dr Ricardo Fernandez is a pulmonary medicine specialist who was involved in the patient care as a consultant.

\section{References}

1 Schneiderman N, Llabre M, Cowie CC, Barnhart J, Carnethon M, Gallo LC, Giachello AL, Heiss G, Kaplan RC, LaVange LM, et al. 2014 Prevalence of diabetes among Hispanics/latinos from diverse backgrounds: The Hispanic Community Health Study/Study of 
Latinos (HCHS/SOL). Diabetes Care 37 2233-2239. (doi:10.2337/ dc13-2939)

2 Lee P-H, Lin H-C, Huang AS-E, Wei S-H, Lai M-S, Lee P-H \& Lin H-H 2014 Diabetes and risk of tuberculosis relapse: nationwide nested case-control study. PLOS ONE 9 e92623. (doi:10.1371/ journal.pone.0092623)

3 Pablos-Méndez A, Blustein J \& Knirsch CA 1997 The role of diabetes mellitus in the higher prevalence of tuberculosis among Hispanics. American Journal of Public Health 87 574. (doi:10.2105/ ajph.87.4.574)

4 Dooley KE \& Chaisson RE 2009 Tuberculosis and diabetes mellitus: convergence of two epidemics. Lancet Infectious Diseases 9 737-746. (doi:10.1016/S1473-3099(09)70282-8)
5 Restrepo BI, Fisher-Hoch SP, Pino PA, Salinas A, Rahbar MH Mora F, Cortes-Penfield N \& McCormick JB 2008 Tuberculosis in poorly controlled type 2 diabetes: altered cytokine expression in peripheral white blood cells. Clinical Infectious Diseases 47634. (doi:10.1086/592125)

6 Koo BK 2013 Diabetes mellitus and tuberculosis. Diabetes and Metabolism Journal 37 249-251. (doi:10.4093/dmj.2013.37.4.249)

7 United States Census Bureau. USA QuickFacts, 8 June 2015. (available at: http://quickfacts.census.gov/qfd/states/00000.html).

8 Centers for Disease Control and Prevention (CDC) 2009 Updated guidelines for the use of nucleic acid amplification tests in the diagnosis of tuberculosis. Morbidity and Mortality Weekly Report 58 7-10.

Received in final form 13 June 2016

Accepted 7 July 2016 